\title{
Potential of zebrafish as a model to characterise MicroRNA profiles in mechanically mediated joint degeneration
}

\author{
Elizabeth A. Lawrence ${ }^{1}$ (D) . Chrissy L. Hammond ${ }^{1}$ (D) $\cdot$ Emma J. Blain $^{2}$ (D)
}

Accepted: 31 August 2020 / Published online: 15 September 2020

(c) The Author(s) 2020

\begin{abstract}
Mechanically mediated joint degeneration and cartilage dyshomeostasis is implicated in highly prevalent diseases such as osteoarthritis. Increasingly, MicroRNAs are being associated with maintaining the normal state of cartilage, making them an exciting and potentially key contributor to joint health and disease onset. Here, we present a summary of current in vitro and in vivo models which can be used to study the role of mechanical load and MicroRNAs in joint degeneration, including: non-invasive murine models of PTOA, surgical models which involve ligament transection, and unloading models based around immobilisation of joints or removal of load from the joint through suspension. We also discuss how zebrafish could be used to advance this field, namely through the availability of transgenic lines relevant to cartilage homeostasis and the ability to accurately map strain through the cartilage, enabling the response of downstream MicroRNA targets to be followed dynamically at a cellular level in areas of high and low strain.
\end{abstract}

Keywords Zebrafish $\cdot$ Joints $\cdot$ Loading $\cdot$ Cartilage $\cdot$ MicroRNA $\cdot$ Osteoarthritis

\section{Introduction}

Skeletal homeostasis is intrinsically linked to mechanical loading, with physiological loading promoting cartilage health (Lee and Bader 1997; Otterness et al. 1998; Soltz et al. 2000; Manninen 2001; Galois et al. 2003; Shelton, Bader and Lee 2003; Sharma, Saxena and Mishra 2007) and maintenance of bone mass (Russo 2009). Abnormal loading of joints, particularly the hip (Croft et al. 1992) and knee (Felson et al. 1991; Coggon et al. 2000), is associated with joint degeneration and osteoarthritis (OA) onset. OA is the most common joint disease globally, with up to $50 \%$ of people over the age of 65 estimated to suffer from the disease (Lawrence et al. 2008; Murphy et al. 2008). During OA, the articular cartilage of joints is degraded leading to ectopic bone formation, joint inflammation and severe pain in sufferers, with almost $75 \%$ of people living with OA experiencing

Elizabeth A. Lawrence

elizabeth.lawrence@bristol.ac.uk

1 School of Physiology, Pharmacology and Neuroscience, University of Bristol, Bristol BS8 1TD, UK

2 Biomechanics and Bioengineering Centre Versus Arthritis, School of Biosciences, Cardiff University, Cardiff CF10 3AX, UK constant pain and $12.5 \%$ describing their pain as frequently unbearable (Arthritis Research UK 2017).

Although changes to mechanical loading have been identified as a major risk factor for OA (Kujala et al. 1995; Lane et al. 1999; McAlindon et al. 1999), the full mechanism by which mechanically mediated joint degeneration occurs during OA onset is not fully understood. It has been suggested that expression of short non-coding MicroRNAs (miRs) which can regulate gene expression and are key in cartilage homeostasis could be key to disease onset. miRs have an average length of 22 nucleotides (O'Brien et al. 2018) and are able to interact with mRNAs, mostly via the 3' untranslated region (UTR), to modify their expression (Fig. 1a) (Ha and Kim 2014). Alterations in articular cartilage miR expression are associated with joint degeneration and OA pathogenesis (Araldi and Schipani 2010; Goldring and Marcu 2012; Swingler et al. 2012); importantly, miRs are reported to be mechanically regulated in cartilage chondrocytes. 
a
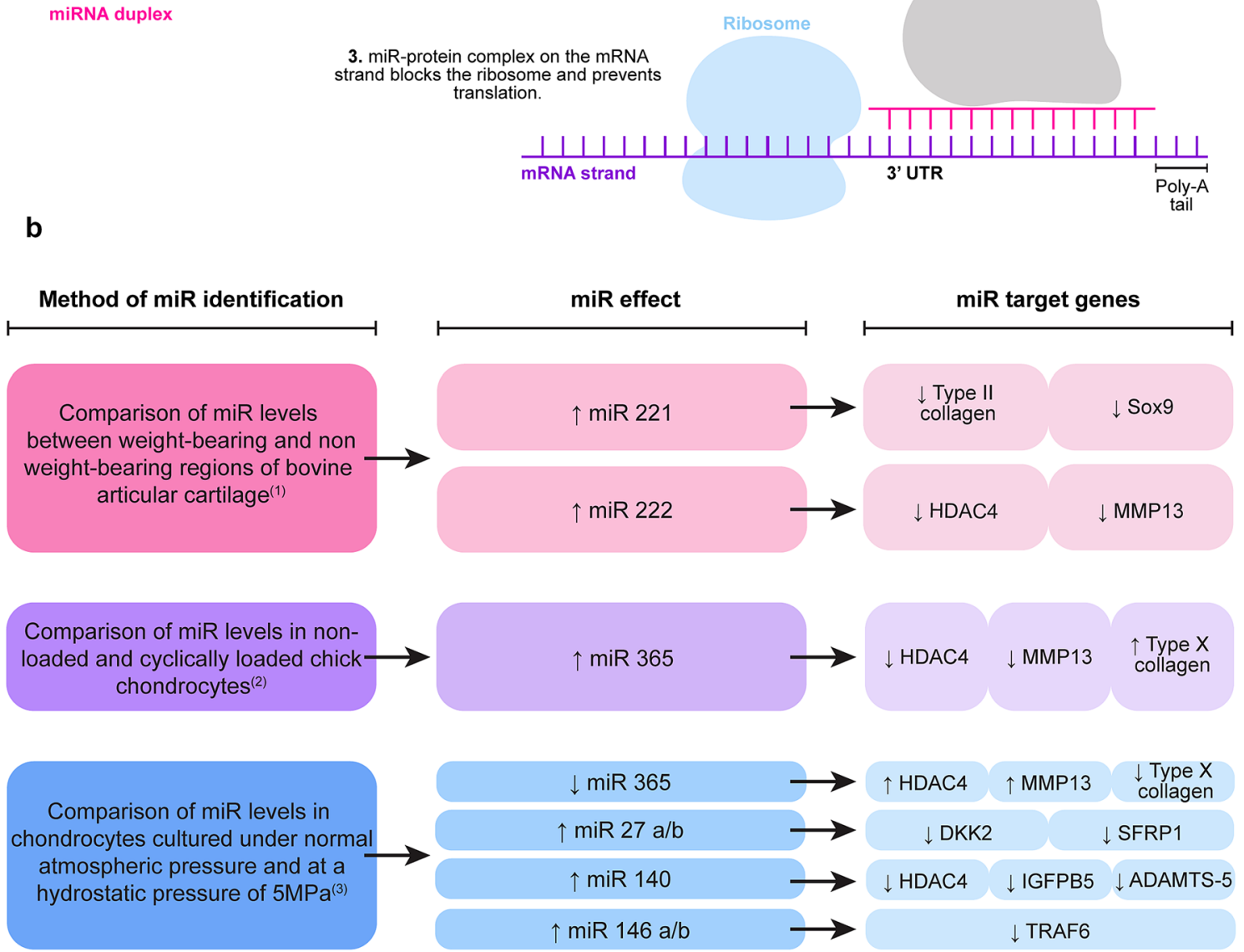

Fig. 1 a Schematic overview of miR interaction with mRNA. b Summary of miRs known to respond to specific stimuli in chondrocytes, and the genes which are affected by changes to these miR expression levels. The mechanical stimulus found to alter miR expression

\section{Mechano-regulation of miRs and their downstream targets in chondrocytes}

Early evidence of mechanically induced epigenetic regulation in articular cartilage was reported in stifle joint medial femoral condyles with increased expression of miR-221 and miR-222 in anterior weight-bearing compared to posterior non-weight-bearing regions (Dunn et al. 2009). Putative gene targets for miR-221 and miR-222 (contains conserved seed site) include histone deacetylase 4 (HDAC4) and matrix metalloproteinase 13 (MMP13) (Song et al. 2015). miR-221 expression inversely correlates with expression of chondrogenic markers including is listed on the left, the miR and how its expression is altered is displayed in the central column and the downstream gene targets of each miR are shown in the right column. ${ }^{(1)}$ Dunn et al. 2009, ${ }^{(2)}$ Guan et al. 2011, ${ }^{(3)}$ Cheleschi et al. 2017

type II collagen and the transcription factor SOX9 in mesenchymal stem cells (Lolli et al. 2014)); thus, it may have relevance in regulating load-induced cartilage homeostasis.

Significant elevation of miR-365 has previously been observed after application of tensile strain to 3D-sponge scaffolds seeded with chick chondrocytes (Guan et al. 2011) and human chondrocytes isolated from macroscopically normal regions of OA cartilage (Yang et al. 2016). A direct target gene is HDAC4 which regulates downstream molecules including MMP13 and type X collagen (Yang et al. 2016).

Significant induction of miR-27a/b, miR-140 and miR$146 \mathrm{a} / \mathrm{b}$ with a concomitant reduction in miR-365 was observed in OA chondrocytes exposed to hydrostatic 
pressure (Cheleschi et al. 2017). Other studies have corroborated the mechano-regulation of miR-146a in chondrocytes (Jin et al. 2014; Guan et al. 2018) and miR-27 (Blain, unpublished observations). WNT signalling molecules e.g. dickkopf-2 (DKK2) and secreted frizzled-related protein 1 (sFRP1) have been identified as miR-27 target genes (Wu et al. 2019), and more recently, the TNF receptor-associated factor-6 (TRAF-6)-mediated NFkB signalling pathway has been shown to be targeted by miR-146 (Shao et al. 2020). miR-140, a key regulator of chondrogenesis and cartilage homeostasis (Miyaki et al. 2010), has many putative target genes, but of relevance to this perspective is its known modulation of HDAC4, insulin growth factor-binding protein 5 (IGFBP5) and a disintegrin and metalloproteinase with thrombospondin motif 5 (ADAMTS-5) (Tuddenham et al. 2006; Cheleschi et al. 2017), all of which are molecules important in maintaining cartilage homeostasis. A summary of mechano-regulated miRs and their downstream targets in chondrocytes can be found in Fig. 1 b.

\section{Existing model systems for investigating involvement of mechanical load in joint degeneration}

Many models have been developed, involving both in vitro and in vivo systems, to investigate the role of abnormal/ altered mechanical load in mediating degeneration of the synovial joint tissues that results in initiation and progression of OA.

\section{In vitro loading models}

Development of in vitro systems for mechanobiology research has utilised isolated cells derived from several species typically including human, mouse, bovine and porcine as either primary or cell lines; mechanical load is subsequently applied to these cells following culture as either a monolayer (Millward-Sadler et al. 2000; Ikenoue et al. 2003) or in 3D constructs (Buschmann et al. 1995; Roberts et al. 2001), or as a co-culture system with at least two different joint cell populations (McCorry, Puetzer and Bonassar 2016). Explant tissues, using only articular cartilage (Guilak et al. 1994) or alternatively an osteochondral plug (cartilage-bone unit) have also been utilised to investigate mechanobiological pathways (Blain et al. 2001; Patwari et al. 2003). There are distinct advantages to using in vitro culture systems to characterise cell behaviour in response to mechanical stimuli. Key amongst these is the ability to accrue a large cell population for experimentation, as well as co-culturing cells of different origins to investigate communication and interplay in response to loading. Use of explant tissue provides an in-situ environment which partially recapitulates an in vivo system due to the presence of an extensive, native extracellular matrix (ECM), facilitating cell-matrix communication, known to be critical in mechano-signalling (Guilak et al. 2006).

Utilisation of these in vitro models has contributed significantly to our understanding of how cells respond to mechanical stimuli and have facilitated characterisation of the molecular pathways that initiate tissue degeneration; however, what they cannot do is recapitulate the complex interactions that exist within the joint tissues, i.e. articular cartilage, bone, meniscus, synovium and associated vasculature. Furthermore, the interplay of mechanical responses with other biological stimuli, e.g. inflammation, or the longitudinal effects of mechanical stimuli on cell/tissue behaviour can only be comprehensively analysed in an in vivo model system.

\section{In vivo loading models}

In vivo loading models have been developed to replicate the pathological features resulting from a joint injury or mechanical insult, often referred to as a model of posttraumatic OA (secondary OA). Such mechanical insult can either be administered surgically, e.g. via damage of the ligaments or meniscus, or via non-invasive means, e.g. application of external loads to the joint.

\section{Surgical loading models}

One of the earliest models that demonstrated the involvement of mechanical injury in induction of joint degeneration and OA pathology was the Pond-Nuki model (Pond and Nuki 1973); transection of the canine anterior cruciate ligament (ACL) altered the stability and biomechanics of the joint resulting in progression of OA. Subsequent studies involving ACL transection in other species including rabbits (Batiste et al. 2004), cats (Herzog et al. 1993) and sheep (Beveridge et al. 2013) have also been performed. Since then, ACL transection in rodent models has been reported with induction of tissue degeneration in response to altered joint biomechanics (Kamekura et al. 2005; Okamura et al. 2010).

Another common surgical approach to studying how joint tissue components respond to altered loading patterns is via meniscal destabilisation involving either partial or complete removal of the medial meniscus, with early studies performed in rabbits (Shapiro and Glimcher 1980) and guinea pigs (Meacock, Bodmer and Billingham 1990), and subsequently in mice (Glasson, Blanchet and Morris 2007). Although these models induce tissue degeneration due to altered joint biomechanics, the procedures themselves are invasive and require opening of the joint capsule; this in itself can initiate an inflammatory response impacting the 
local environment and may not be completely representative of the mechanical insult alone, thus necessitating increased animal numbers to provide "sham" controls (incision only surgeries).

\section{Non-invasive loading models}

In recent years, there has been an emergence in non-invasive mouse models of post-traumatic OA (PTOA). Such models are believed to more accurately recapitulate the mechanisms involved in mechanically induced injuries in humans, initiating joint degeneration through direct damage to the tissue components of the joint. These non-invasive models rely on the external application of a mechanical load to the tibia, either as a single traumatic insult or a defined period of loading, without any surgical intervention.

Utilisation of a single compressive load (12 N) has been previously demonstrated to rupture the ACL initiating pathological changes in murine articular cartilage and underlying subchondral bone (Christiansen et al. 2012). Pathologically distinct phases with an early inflammatory phase and a later degenerative component are evident in the non-invasive murine PTOA model (Gilbert et al. 2018); this proves advantageous as a model system as it provides a 'therapeutic window' following mechanical insult in which to assess treatments to delay or halt joint degeneration.

Tibial compression, via the application of cyclic axial compressive load transmitted through the natural articulation of the murine knee joint, induces articular cartilage overloading and tissue damage, following multiple loading episodes (Poulet et al. 2011). These non-invasive loading models have also been used to investigate the interplay of mechanical load and genetics, with use of genetically engineered mice (gene knockouts and transgenic overexpression) to more clearly delineate the importance of these risk factors in joint degeneration. Overall, the non-invasive loading models obviate the need for technically challenging surgical techniques, but more importantly avoid any confounding effects induced by the trauma of the surgical procedure and are more translatable to mechanical trauma experienced by humans.

\section{Models of unloading}

The absence of weight-bearing is equally detrimental to the health of the joint tissues demonstrating that a physiological range of mechanical load is essential for maintenance of homeostasis. Numerous in vivo models of joint 'unloading' or immobilisation have been utilised to characterise how the absence of loading affects tissue behaviour, particularly in articular cartilage. Seminal early studies induced knee joint immobilisation using casts or surgery (limb amputation), typically in canine models (Palmoski, Perricone and Brandt
1979; Palmoski, Colyer and Brandt 1980), rabbits (Sood 1971; Langenskiöld, Michelsson and Videman 1979) and later in rodents (Hagiwara et al. 2009). An alternative model involving suspension of rodents by the tail has also been used to recapitulate hindlimb unloading (Tomiya et al. 2009; Nomura et al. 2017). Joint unloading inhibited expression of key ECM components, e.g. proteoglycans, reduced cartilage thickness and induced surface fibrillations, resulting in disruption of articular cartilage integrity. Interestingly, effects were reversible following joint 'reuse' and the reintroduction of weight-bearing (Palmoski, Perricone and Brandt 1979; Behrens, Kraft and Oegema 1989), demonstrating the highly adaptive nature of articular cartilage.

\section{Prospects for using zebrafish as a model for mechanically mediated joint degeneration to investigate $\mathrm{miR}$ dysregulation}

Increasingly, the freshwater teleost zebrafish is used as a laboratory model for disease with the low cost of housing, ease of genetic manipulation, rapid development (which has been carefully staged (Kimmel et al. 1995)) and genetic tractability among the major advantages of this model. Although zebrafish are more remote from humans than other animal models (last common ancestor was 445 million years ago compared to 96 million years ago for rodents (Ali et al. 2011)) and the fact they have undergone genome duplication (Meyer and Schartl 1999): many genes are conserved (Dodd et al. 2000; Taylor et al. 2003) with around $70 \%$ of human genes found to have at least one orthologue (Howe et al. 2013) and $85 \%$ of disease-related genes being conserved (Wellcome Trust 2013) in zebrafish. Generally, zebrafish are ideal model organisms for studying disease as they have transparent larvae which enable dynamic longitudinal in vivo imaging in the same fish throughout maturation. They have high fecundity with the eggs developing externally, meaning the mother does not have to be killed to study early development, thus reducing animal costs and complying with the $3 \mathrm{Rs}$ principles of reduction and replacement (Russell and Burch 1959). In addition to these more general advantages, a number of specific factors are discussed below which make zebrafish an attractive prospect for studies into the role of miRs in mechanically mediated joint degeneration.

\section{Zebrafish cartilage contains components also found in human articular cartilage. Loading of cartilage can be manipulated in zebrafish}

At 5 days post fertilisation (dpf) the zebrafish craniofacial skeleton is made up of distinct cartilaginous elements 

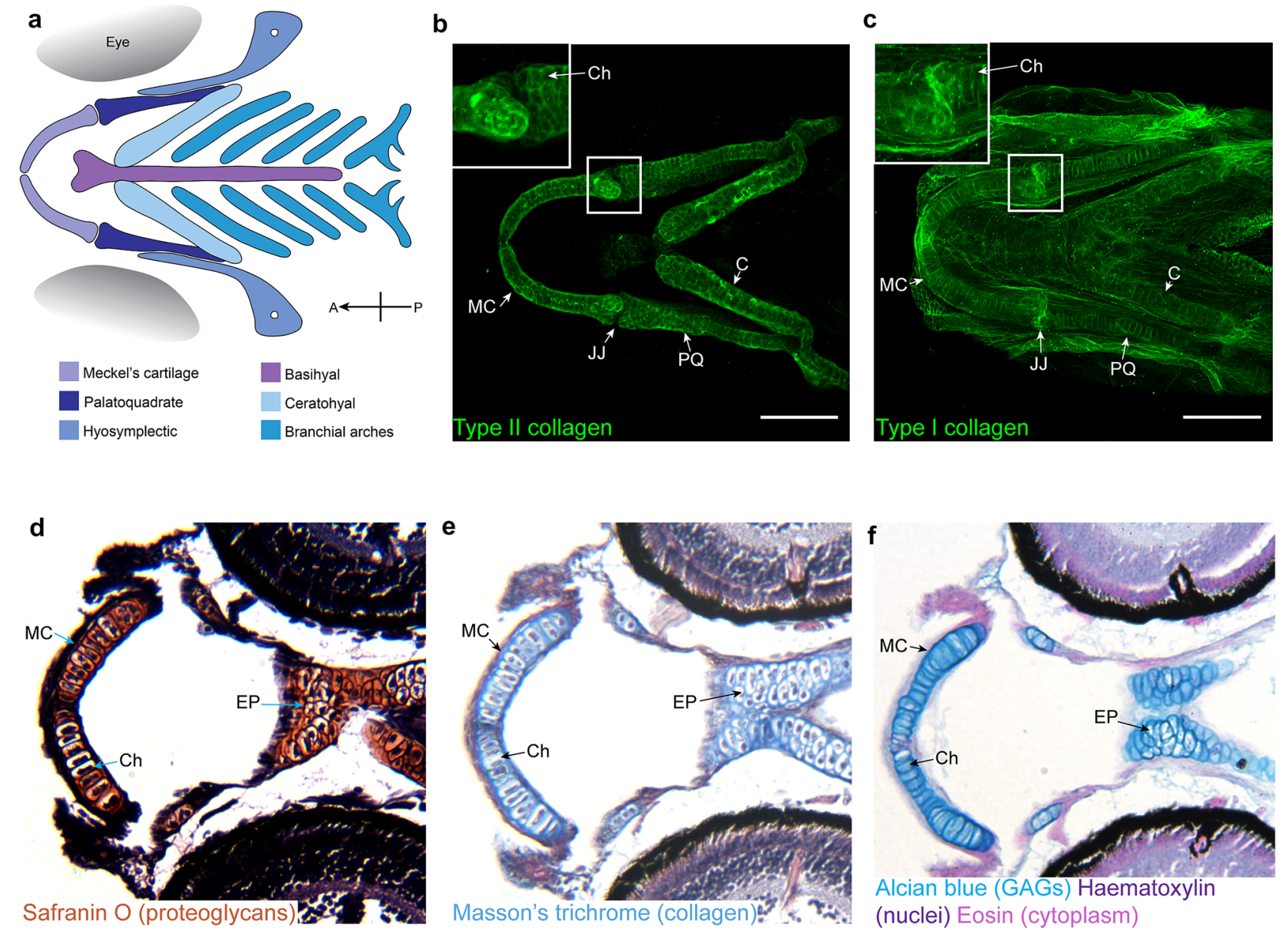

Fig. 2 Zebrafish craniofacial cartilage has key components found in human articular cartilage. a Schematic representation of the cartilage elements which make up the zebrafish craniofacial skeleton at $5 \mathrm{dpf}$. Orientation compass included in bottom right, with $A$ anterior and $P$ posterior. b, c Ventral view confocal image stacks of $5 \mathrm{dpf}$ craniofacial skeleton immunostained for type II collagen (b) and type I collagen (c) in the ECM surrounding chondrocytes. Inset shows zoom of jaw joint. Scale $\mathrm{bar}=100 \mu \mathrm{m}$. Images in $\mathrm{b}$ and $\mathrm{c}$ previously published

(Fig. 2a) derived from the migratory neural crest (Schilling and Kimmel 1994). This cartilage contains all the key components of human cartilage including chondrocytes which undergo hypertrophy (Mitchell et al. 2013; Askary et al. 2016), collagens and proteoglycans (Fig. 2b-f).

As with human cartilage, the craniofacial cartilages of larval zebrafish have been shown to be mechanically sensitive (Brunt et al. 2016). The biomechanical load exerted on the craniofacial cartilages and joint can be controlled in larvae genetically or pharmacologically to induce paralysis (either flaccid or rigid) to reduce load, or the induction of hyperactivity to induce excessive loading conditions (Shwartz et al. 2012; Brunt et al. 2016). Strains in the zebrafish skeleton, as in other systems, can be modelled computationally using Finite Element Analysis (FEA) (Fig. 3h), a computational in (Lawrence et al. 2018). d-f Ventral paraffin sections of 5 dpf craniofacial skeleton stained with Safranin O (d) to show proteoglycans, Masson's trichrome (e) to show the presence of collagen, and alcian blue (f) to show the presence of glycosaminoglycans in the chondrocyte ECM which makes up the cartilage. $M C$ Meckel's cartilage, $J J$ jaw joint, $P Q$ palatoquadrate, $C$ ceratohyal, $C h$ example of a chondrocyte surrounded by ECM, EP ethmoid plate

technique that predicts deformation, stress and strain in a structure when subjected to external loading conditions (Bright and Rayfield 2011). FEA can be used to visualise patterns of strain by displaying them with colours to show areas of high and low strain, or degree of tissue deformation. Finite element models for the developing zebrafish jaw have been published for wild-type fish (Brunt et al. 2016; Brunt et al. 2016), mutants (Lawrence et al. 2018) and larvae exposed to different gravitational fields (Lawrence et al. 2020). Cartilage material properties change during development, in response to mutations to chondrocyte genes or changes to gravitational force, and can be tested ex vivo by atomic force microscopy (AFM) or nanoindentation (Lawrence et al. 2018, 2020), to provide further information and accuracy to FE models. 
a

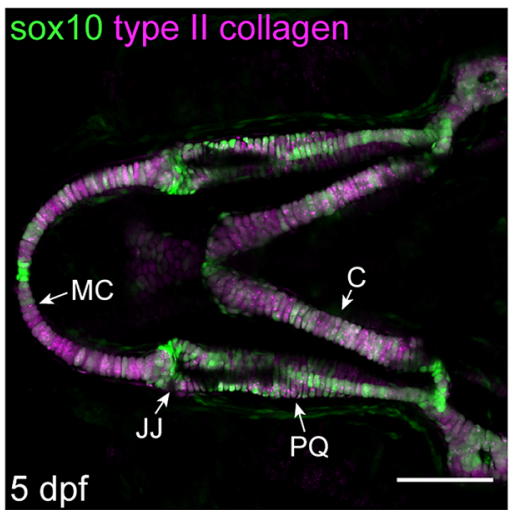

d'

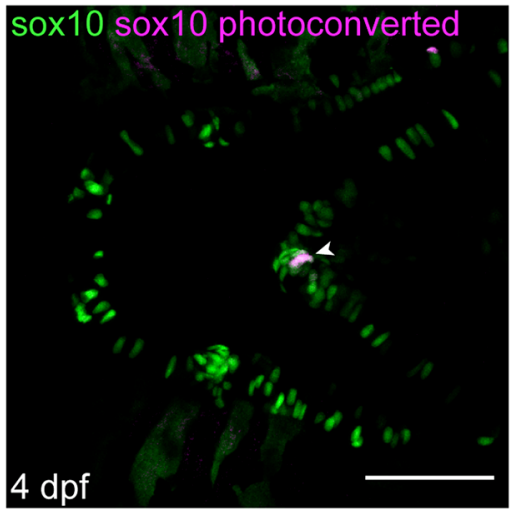

f
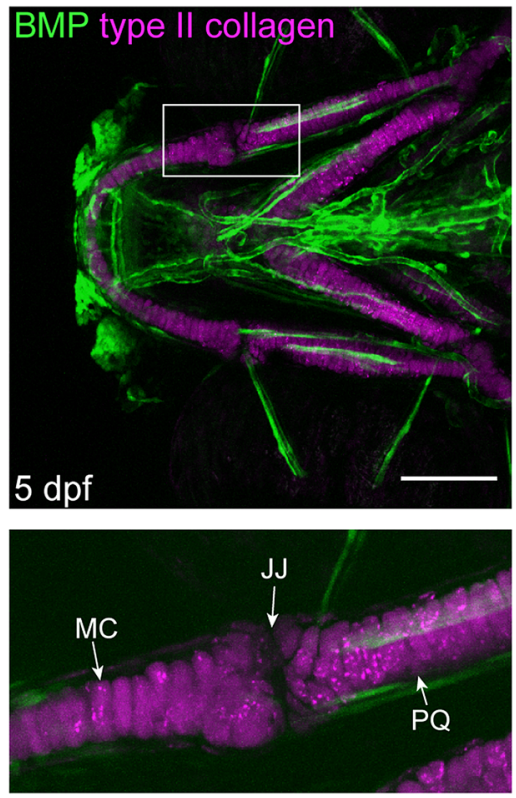

b

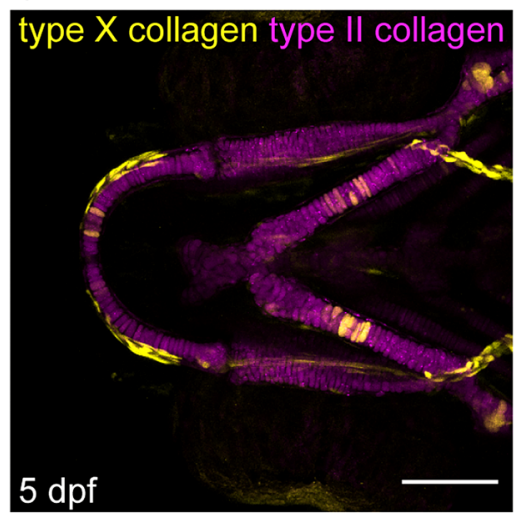

d"

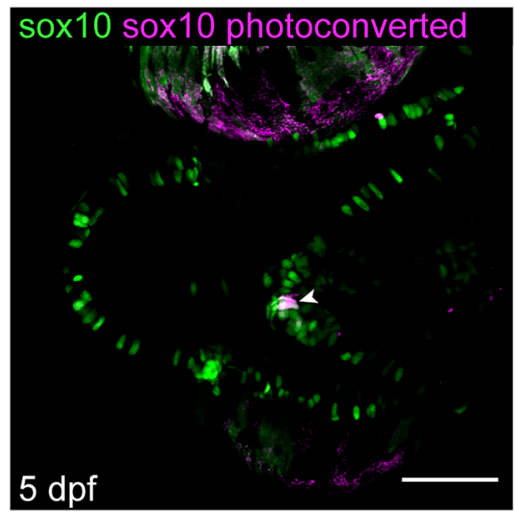

g
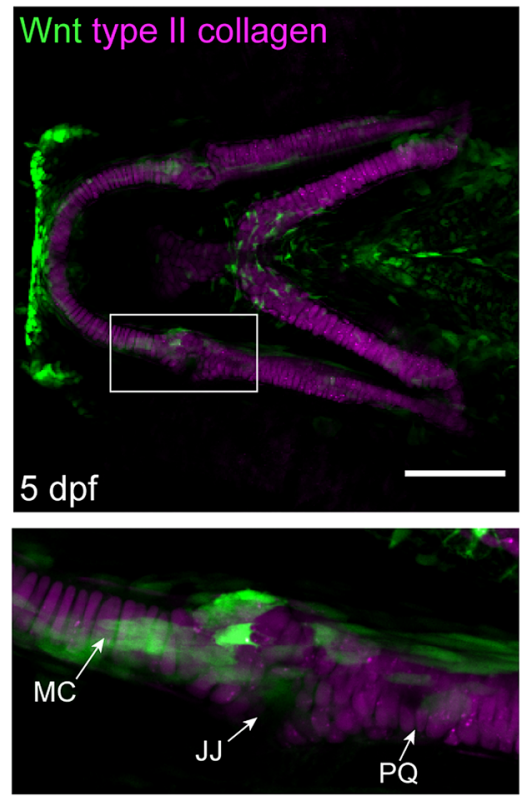

c

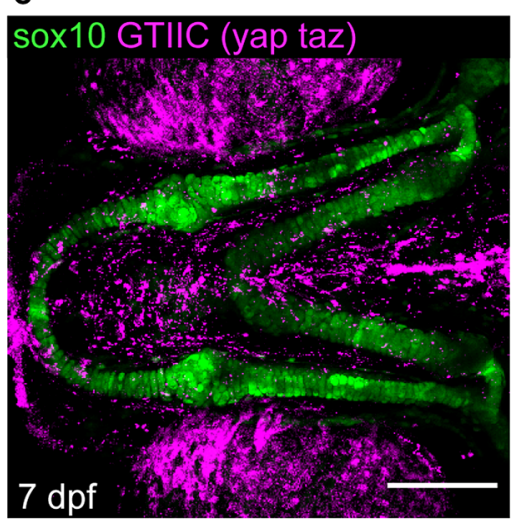

e'

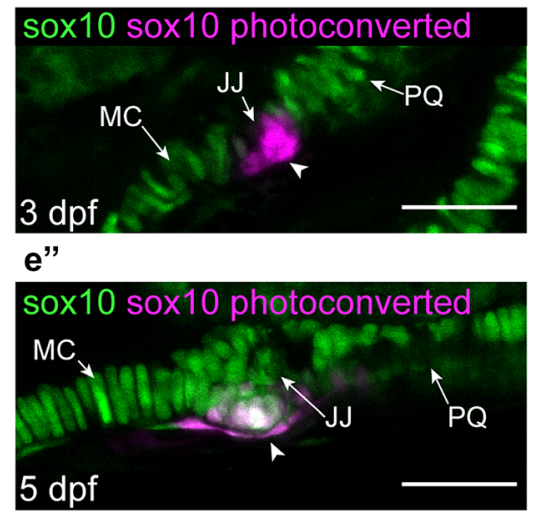

h
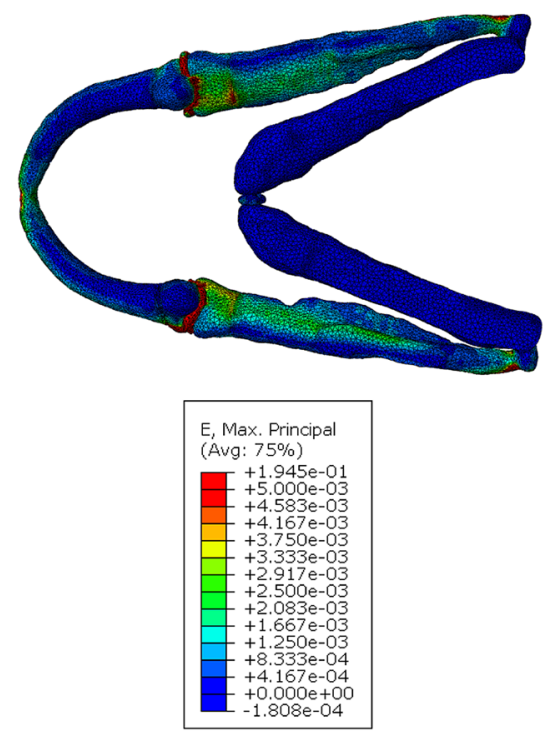

Cavitation of the jaw joint between the Meckel's cartilage and the palatoquadrate to form a fluid-filled synovium (as opposed to a cellular interzone) occurs late in zebrafish (around $14 \mathrm{dpf}$ ) relative to onset of joint movement; this joint continues to mature, eventually forming a synovial joint with all of the tissues observed in a human synovial joint (Askary et al. 2016). As the cartilaginous craniofacial skeleton forms early in development and many elements are 
4Fig. 3 Tools available in zebrafish to facilitate spatiotemporal study of miRNA targets at a cellular level. a-c Ventral view confocal images of the craniofacial cartilages of Tg(4.9Sox 10:EGFP; col2a 1aBAC:mcherry) (a), Tg(col2alaBAC:mcherry;colloalaBAC:citr ine)hu7050 (b), Tg(- 4.9Sox10:EGFP; 4xGTIIC:eGFP) (c) imaged live. Scale bar $=100 \mu \mathrm{m}$. d', d' Ventral view confocal images of the craniofacial cartilages of $\mathrm{Tg}$ (Sox10:GAL4-VP16; UAS:Kaede) with photoconverted chondrocytes shown in magenta and annotated with a white arrowhead in the same fish at $4 \mathrm{dpf}(\mathbf{d}$ ') and $5 \mathrm{dpf}(\mathbf{d}$ '). Scale bar $=100 \mu \mathrm{m}$. e'-e'" Confocal images of the lower jaw joint in Tg(Sox10:GAL4-VP16; UAS:Kaede) with photoconverted chondrocytes shown in magenta and annotated with a white arrowhead in the same fish at $3 \mathrm{dpf}\left(\mathbf{e}^{\prime}\right)$ and $5 \mathrm{dpf}$ (e'"). Scale bar=50 $\mu \mathrm{m}$, images in e' and e" previously published in (Brunt et al. 2017). f, g Ventral view confocal images of the craniofacial cartilages of $\operatorname{Tg}(5 x B M P R E-X l a$. Id3:GFP; col2alaBAC:mCherry) (f) and Tg(7xTCF.XlaSiam:nlsGFP ;col2alaBAC:mCherry) (g) imaged live. Scale bar $=100 \mu \mathrm{m}$. h Finite element model of maximum principal (E. max) strains in 5dpf wildtype zebrafish viewed from a dorsal orientation. E. max represents tensional strains with cooler colours (blue) on the scale corresponding to lower strain and red corresponding to higher strain values. $M C$ Meckel's cartilage, $J J$ jaw joint, $P Q$ palatoquadrate, $C$ ceratohyal

retained into adulthood, it is a valuable model to investigate at a cellular level how the cartilage is affected by different genetic mutations, such as those to downstream miR targets, and environmental factors, such as mechanical loading.

The presence of lower jaw cartilages in which loading can be manipulated allows miRs which are responsive to mechanical load to be isolated (Chen et al. 2005) and identified so that specific pathways can be dysregulated through CRISPR/cas9-induced mutation of miR signalling pathway components or through immersion in miR agonists/antagonists and the effect on chondrocytes studied. Other skeletal elements in zebrafish, such as the vertebral centra, have also been demonstrated to be mechanically sensitive, and to undergo remodelling in response to load (Fiaz et al. 2012; Ofer et al. 2019), which could be regulated by miRs. Finite element models also exist for the zebrafish vertebral column (Newham et al. 2019; Ofer et al. 2019). However, as patterning and mineralisation of the vertebral column occur somewhat later than that of the jaw (from $8 \mathrm{dpf}$ onwards) (Wopat et al. 2018), testing the role of specific miRs in the vertebral column is likely to be more technically challenging.

\section{Tools available in zebrafish which allow for cellular changes to be observed in the context of the whole tissue}

A major advantage to zebrafish as a model is the possibility to dynamically image cellular events which could be impacted by miR dysregulation such as chondrocyte behaviour, ECM production and the expression of targets downstream of the mechanically regulated $\mathrm{miR}$ in the living zebrafish. This can be achieved through high-resolution confocal or lightsheet imaging of transgenic reporter lines.
Many transgenic lines (listed in full in Table 1) relevant to the study of cartilage homeostasis and degeneration exist, including lines which facilitate the study of chondrocyte behaviour and expression of important ECM components such as the sox9:eGFP, sox10:eGFP, col2:mCherry and colx: citrine lines. As discussed, these molecules have been implicated as downstream miR targets. In the context of zebrafish, they enable chondrocyte morphology and migration within the cartilage to be tracked throughout maturation from immature stages when sox 9 and sox 10 are predominantly expressed (Fig. 3a), to hypertrophic stages just prior to ossification when chondrocytes express $\operatorname{colX}$ (Schmid et al. 1991; Mitchell et al. 2013);(Fig. 3b). In later larval stages, the impact of load on osteogenesis can be studied dynamically, for example with the GTIIC:eGFP line which shows Yap/Taz - TEAD activity (Fig. 3c). Photoconvertible transgenics such as sox 10:kaede offer the opportunity to track single cells (Fig. 3d'-e') to study how their migration and maturation are affected depending on their location in the cartilage and the forces they are exposed to.

Zebrafish represent a model in which the spatiotemporal response of both cells and genes to a mechanical stimuli can be measured simultaneously. Finite Element modelling can be used to measure the response of specific cell populations, with areas of altered strain having been mapped previously (Fig. 3h) and changes to chondrocyte morphology and ECM composition in these regions analysed (Lawrence et al. 2020). Live imaging of transgenic reporter lines such as the wnt:GFP or the BMP:GFP reporter (Fig. 3f, g) also enables the response of specific genes to be tracked in regions of abnormal strain. This technique was used to correlate regions of high strain in joint morphogenesis with Wnt reporter expression, leading to the identification of Wnt 16 as a mechanoresponsive gene that controls joint cell behaviour (Brunt et al. 2017). This allows the effects of a genetic mutation or pharmacological intervention to be mapped according to where in the cartilage a chondrocyte is, for example at the joint or at a muscle attachment site, to see if some populations of cells react differently to stimuli.

In addition to the transgenic lines available, there is also good availability of miR tools in zebrafish to enable study of joint degeneration in the context of mechanically mediated miRs. miRs have been shown to play a role in cartilage development and homeostasis in zebrafish. Zebrafish of the dicerl mutant line, which lacks the Dicer miR processing enzyme, have abnormal craniofacial development and increased expression of sox 10 (Weiner et al. 2019), confirming that miRs are essential for normal development (Wienholds et al. 2003). A number of miRs such as miR-140 and miR-29 are regulated by sox 9 in zebrafish, and in turn control aspects of chondrocyte behaviour (Nakamura et al. 2012; Le et al. 2016), while others such as miR-92a maintain BMP signalling in cartilage. The spatiotemporal expression 
Table 1 Full nomenclature of transgenic lines important for study of mechanically mediated joint degeneration

\begin{tabular}{|c|c|c|}
\hline Text reference & Full transgenic name & References \\
\hline sox9:eGFP & $\operatorname{Tg}\left(-2421 /+29 \operatorname{sox} 9 b: E G F P_{\mathrm{uw} 2}\right)$ & Garcia et al. (Garcia et al. 2017) \\
\hline sox10:eGFP & $\operatorname{Tg}(-4.9$ Sox $10: E G F P)$ & Wada et al. (Wada et al. 2005) \\
\hline col2:mCherry & $\operatorname{Tg}($ col2alaBAC:mcherry) & $\begin{array}{l}\text { Hammond and Schulte-Merker (Hammond and } \\
\text { Schulte-Merker 2009) }\end{array}$ \\
\hline colx:citrine & $\operatorname{Tg}($ collOalaBAC:citrine $) h u 7050$ & Mitchell et al. (Mitchell et al. 2013) \\
\hline GTIIC:eGFP & $\operatorname{Tg}(4 x G T I I C: E G F P)$ & Miesfield and Link 2014 (Miesfeld and Link 2014) \\
\hline sox10:kaede & $\operatorname{Tg}($ Sox10:GAL4-VP16;UAS:Kaede) & Hatta et al. (Hatta, Tsujii and Omura 2006) \\
\hline$w n t: G F P$ & $\operatorname{Tg}(7 x T C F . X l a S i a m: n l s G F P)$ & Moro et al. (Moro et al. 2012) \\
\hline$B M P: G F P$ & $\operatorname{Tg}(5 x B M P R E-X l a . I d 3: G F P)$ & Alexander et al. (Alexander et al. 2011) \\
\hline
\end{tabular}

of a number of miRs in zebrafish has also been determined using techniques such as in-situ hybridisation and microarrays (Wienholds et al. 2005). This includes the mapping of 115 miRs which are conserved from vertebrates (Wienholds et al. 2005).

Synthetic miRs have successfully been used to downregulate genes of interest in zebrafish (Giacomotto, Rinkwitz and Becker 2015), indicating that this model system is tolerant to miR injection to target downstream molecules. This would enable specific miR signalling pathways to be inhibited in existing transgenic lines and the resulting changes to cells in areas of known strain to be studied dynamically.

We conclude that despite the existence of several model systems to study the role of mechanical loading in joint degeneration, and the emergence of non-invasive mouse models of PTOA, zebrafish offer the advantage of live deep skeletal tissue imaging in response to changes in load or inhibition of miR signalling.

Acknowledgements The authors would like to acknowledge the Biomechanics and Bioengineering Research Centre Versus Arthritis (510390) at Cardiff University and the Wolfson Bioimaging Facility at the University of Bristol. They would also like to thank Roddy Skinner for the image in Fig. 3c and Jessye Aggleton for the Finite Element models shown in Fig. 3h.

Funding EL is funded by Wellcome Trust Dynamic Molecular Cell Biology Doctoral training studentship. $\mathrm{CH}$ is funded by Versus Arthritis Senior Fellowship 21937.

Open Access This article is licensed under a Creative Commons Attribution 4.0 International License, which permits use, sharing, adaptation, distribution and reproduction in any medium or format, as long as you give appropriate credit to the original author(s) and the source, provide a link to the Creative Commons licence, and indicate if changes were made. The images or other third party material in this article are included in the article's Creative Commons licence, unless indicated otherwise in a credit line to the material. If material is not included in the article's Creative Commons licence and your intended use is not permitted by statutory regulation or exceeds the permitted use, you will need to obtain permission directly from the copyright holder. To view a copy of this licence, visit http://creativecommons.org/licenses/by/4.0/.

\section{References}

Alexander $\mathrm{C}$ et al (2011) Combinatorial roles for BMPs and endothelin 1 in patterning the dorsal-ventral axis of the craniofacial skeleton. Development 138(23):5135-5146. https://doi.org/10.1242/ dev.067801

Ali S et al (2011) Zebrafish embryos and larvae: a new generation of disease models and drug screens. Birth Defects Res C Embryo Today 93(2):115-133. https://doi.org/10.1002/bdrc.20206

Araldi E, Schipani E (2010) MicroRNA-140 and the silencing of osteoarthritis. Genes Dev 24(11):1075-1080. https://doi.org/10.1101/ gad.1939310

Arthritis Research UK (2017) State of Musculoskeletal Health 2017.

Askary A et al (2016) Ancient origin of lubricated joints in bony vertebrates. Elife 5:e16415. https://doi.org/10.7554/eLife.16415

Batiste DL et al (2004) Ex vivo characterization of articular cartilage and bone lesions in a rabbit ACL transection model of osteoarthritis using MRI and micro-CT. Osteoarthritis Cartilage 12(12):986-996. https://doi.org/10.1016/j.joca.2004.08.010

Behrens F, Kraft EL, Oegema TR (1989) Biochemical changes in articular cartilage after joint immobilization by casting or external fixation. J Orthop Res 7(3):335-343. https://doi. org/10.1002/jor.1100070305

Beveridge JE et al (2013) Tibiofemoral centroid velocity correlates more consistently with cartilage damage than does contact path length in two ovine models of stifle injury. J Orthop Res 31(11):1745-1756. https://doi.org/10.1002/jor.22429

Blain EJ et al (2001) Up-regulation of matrix metalloproteinase expression and activation following cyclical compressive loading of articular cartilage in vitro. Arch Biochem Biophys 396(1):49-55. https://doi.org/10.1006/abbi.2001.2575

Bright JA, Rayfield EJ (2011) The response of cranial biomechanical finite element models to variations in mesh density. Anat Rec 294(4):610-620. https://doi.org/10.1002/ar.21358

Brunt L et al (2016) Building finite element models to investigate zebrafish jaw biomechanics. J Vis Exp. https://doi. org/10.3791/54811

Brunt LH et al (2016) Differential effects of altered patterns of movement and strain on joint cell behaviour and skeletal morphogenesis. Osteoarthritis Cartilage 24(11):1940-1950. https:// doi.org/10.1016/J.JOCA.2016.06.015

Brunt LH et al (2017) Wnt signalling controls the response to mechanical loading during zebrafish joint development. Development 144(15):2798-2809. https://doi.org/10.1242/dev.15352 8

Buschmann MD et al (1995) Mechanical compression modulates matrix biosynthesis in chondrocyte/agarose culture. J Cell Sci 108(4):1497-1508 
Cheleschi S et al (2017) Hydrostatic pressure regulates MicroRNA expression levels in osteoarthritic chondrocyte cultures via the Wnt//-catenin pathway. Int J Mol Sci 18(1):133. https://doi. org/10.3390/ijms 18010133

Chen PY et al (2005) The developmental miRNA profiles of zebrafish as determined by small RNA cloning. Genes Dev 19(11):1288-1293. https://doi.org/10.1101/gad.1310605

Christiansen BA et al (2012) Musculoskeletal changes following non-invasive knee injury using a novel mouse model of posttraumatic osteoarthritis. Osteoarthritis Cartilage 20(7):773782. https://doi.org/10.1016/j.joca.2012.04.014

Coggon D et al (2000) Occupational physical activities and osteoarthritis of the knee. Arthritis Rheum 43(7):1443-1449. https:// doi.org/10.1002/1529-0131(200007)43:7

Croft P et al (1992) Osteoarthritis of the hip and occupational activity. Scand J Work Environ Health 18(1):59-63. https://doi. org/10.5271/sjweh. 1608

Dodd A et al (2000) Zebrafish: bridging the gap between development and disease. Hum Mol Genet 9(16):2443-2449. https:// doi.org/10.1093/hmg/9.16.2443

Dunn W, DuRaine G, Reddi AH (2009) Profiling microRNA expression in bovine articular cartilage and implications for mechanotransduction. Arthritis Rheum 60(8):2333-2339. https://doi. org/10.1002/art.24678

Felson DT et al (1991) Occupational physical demands, knee bending, and knee osteoarthritis: results from the Framingham Study. J Rheumatol 18(10):1587-1592

Fiaz AW et al (2012) Swim-training changes the spatio-temporal dynamics of skeletogenesis in zebrafish larvae (danio rerio). PLoS ONE 7(4):e34072. https://doi.org/10.1371/journ al.pone. 0034072

Galois L et al (2003) Moderate-impact exercise is associated with decreased severity of experimental osteoarthritis in rats. Rheumatology 42(5):692-693. https://doi.org/10.1093/rheumatolo gy/keg094

Garcia GR et al (2017) In vivo characterization of an AHR-dependent long noncoding RNA required for proper Sox 9b expression. Mol Pharmacol 91(6):609-619. https://doi.org/10.1124/ mol.117.108233

Giacomotto J, Rinkwitz S, Becker TS (2015) Effective heritable gene knockdown in zebrafish using synthetic microRNAs. Nat Commun 6(1):1-11. https://doi.org/10.1038/ncomms8378

Gilbert SJ et al (2018) Inflammatory and degenerative phases resulting from anterior cruciate rupture in a non-invasive murine model of post-traumatic osteoarthritis. J Orthop Res 36(8):2118-2127. https://doi.org/10.1002/jor.23872

Glasson SS, Blanchet TJ, Morris EA (2007) The surgical destabilization of the medial meniscus (DMM) model of osteoarthritis in the 129/SvEv mouse. Osteoarthritis Cartilage 15(9):10611069. https://doi.org/10.1016/j.joca.2007.03.006

Goldring MB, Marcu KB (2012) Epigenomic and microRNA-mediated regulation in cartilage development, homeostasis, and osteoarthritis. Trends Mol Med 18(2):109-118. https://doi. org/10.1016/j.molmed.2011.11.005

Guan Y et al (2011) MiR-365: a mechanosensitive microRNA stimulates chondrocyte differentiation through targeting histone deacetylase 4. FASEB J 25(12):4457-4466. https://doi. org/10.1096/fj.11-185132

Guan YJ et al (2018) Evidence that miR-146a attenuates aging- and trauma-induced osteoarthritis by inhibiting Notch 1 , IL-6, and IL-1 mediated catabolism. Aging Cell 17(3):e12752. https:// doi.org/10.1111/acel.12752

Guilak F et al (1994) The effects of matrix compression on proteoglycan metabolism in articular cartilage explants. Osteoarthritis Cartilage 2(2):91-101. https://doi.org/10.1016/S1063 $-4584(05) 80059-7$
Guilak F et al (2006) The pericellular matrix as a transducer of biomechanical and biochemical signals in articular cartilage. Ann N Y Acad Sci 1068:498-512

Ha M, Kim VN (2014) Regulation of microRNA biogenesis. Nat Rev Mol Cell Biol 15(8):509-524. https://doi.org/10.1038/nrm3838

Hagiwara Y et al (2009) Changes of articular cartilage after immobilization in a rat knee contracture model. J Orthop Res 27(2):236-242. https://doi.org/10.1002/jor.20724

Hammond CL, Schulte-Merker S (2009) Two populations of endochondral osteoblasts with differential sensitivity to Hedgehog signalling. Development 136(23):3991-4000. https://doi. org/10.1242/dev.042150

Hatta K, Tsujii H, Omura T (2006) Cell tracking using a photoconvertible fluorescent protein. Nat Protoc 1(2):960-967. https://doi. org/10.1038/nprot.2006.96

Herzog W et al (1993) Hindlimb loading, morphology and biochemistry of articular cartilage in the ACL-deficient cat knee. Osteoarthritis Cartilage 1(4):243-251. https://doi.org/10.1016/S1063 $-4584(05) 80330-9$

Howe K et al (2013) The zebrafish reference genome sequence and its relationship to the human genome. Nature 496(7446):498-503. https://doi.org/10.1038/nature12111

Ikenoue $\mathrm{T}$ et al (2003) Mechanoregulation of human articular chondrocyte aggrecan and type II collagen expression by intermittent hydrostatic pressure in vitro. J Orthop Res 21(1):110-116. https ://doi.org/10.1016/S0736-0266(02)00091-8

Jin L et al (2014) Role of miR-146a in human chondrocyte apoptosis in response to mechanical pressure injury in vitro. Int J Mol Med 34(2):451-463. https://doi.org/10.3892/ijmm.2014.1808

Kamekura $S$ et al (2005) Osteoarthritis development in novel experimental mouse models induced by knee joint instability. Osteoarthritis Cartilage 13(7):632-641. https://doi.org/10.1016/j. joca.2005.03.004

Kimmel CB et al (1995) Stages of embryonic development of the zebrafish. Dev Dyn 203(3):253-310. https://doi.org/10.1002/ aja.1002030302

Kujala UM et al (1995) Knee osteoarthritis in former runners, soccer players, weight lifters, and shooters. Arthritis Rheum 38(4):539 546. https://doi.org/10.1002/art.1780380413

Lane NE et al (1999) Recreational physical activity and the risk of osteoarthritis of the hip in elderly women. J Rheumatol 26(4):849-854

Langenskiöld A, Michelsson JE, Videman T (1979) Osteoarthritis of the knee in the rabbit produced by immobilization: attempts to achieve a reproducible model for studies on pathogenesis and therapy. Acta Orthop 50(1):1-14. https://doi.org/10.3109/17453 677909024083

Lawrence EA et al (2018) The mechanical impact of col11a2 loss on joints; col11a2 mutant zebrafish show changes to joint development and function, which leads to early-onset osteoarthritis. Philos Trans R Soc B Biol Sci 373(1759):20170335. https://doi. org/10.1098/rstb.2017.0335

Lawrence EA et al (2020) Exposure to hypergravity during zebrafish development alters cartilage material properties and strain distribution. bioRxiv. https://doi.org/10.1101/2020.05.26.116046

Lawrence RC et al (2008) Estimates of the prevalence of arthritis and other rheumatic conditions in the United States. Part II. Arthritis Rheum 58(1):26-35. https://doi.org/10.1002/art.23176

Le LTT et al (2016) The microRNA-29 family in cartilage homeostasis and osteoarthritis. J Mol Med 94(5):583-596. https://doi. org/10.1007/s00109-015-1374-z

Lee DA, Bader DL (1997) Compressive strains at physiological frequencies influence the metabolism of chondrocytes seeded in agarose. J Orthop Res 15(2):181-188. https://doi.org/10.1002/ jor.1100150205 
Lolli A et al (2014) Pro-chondrogenic effect of miR-221 and slug depletion in human MSCs. Stem Cell Rev Rep 10(6):841-855. https://doi.org/10.1007/s12015-014-9532-1

Manninen P (2001) Physical exercise and risk of severe knee osteoarthritis requiring arthroplasty. Rheumatology 40(4):432-437. https://doi.org/10.1093/rheumatology/40.4.432

McAlindon TE et al (1999) Level of physical activity and the risk of radiographic and symptomatic knee osteoarthritis in the elderly: the Framingham study. Am J Med 106(2):151-157. https://doi. org/10.1016/s0002-9343(98)00413-6

McCorry MC, Puetzer JL, Bonassar LJ (2016) Characterization of mesenchymal stem cells and fibrochondrocytes in three-dimensional co-culture: analysis of cell shape, matrix production, and mechanical performance. Stem Cell Res Ther 7(1):39. https:// doi.org/10.1186/s13287-016-0301-8

Meacock SC, Bodmer JL, Billingham ME (1990) Experimental osteoarthritis in guinea-pigs. J Exp Pathol 71(2):279-293

Meyer A, Schartl M (1999) Gene and genome duplications in vertebrates: the one-to-four (-to-eight in fish) rule and the evolution of novel gene functions. Curr Opin Cell Biol 11(6):699-704. https ://doi.org/10.1016/s0955-0674(99)00039-3

Miesfeld JB, Link BA (2014) Establishment of transgenic lines to monitor and manipulate Yap/Taz-Tead activity in zebrafish reveals both evolutionarily conserved and divergent functions of the Hippo pathway. Mech Dev 133:177-188. https://doi. org/10.1016/j.mod.2014.02.003

Mitchell RE et al (2013) New tools for studying osteoarthritis genetics in zebrafish. Osteoarthritis Cartilage 21(2):269-278. https://doi. org/10.1016/j.joca.2012.11.004

Miyaki S et al (2010) MicroRNA-140 plays dual roles in both cartilage development and homeostasis. Genes Dev 24(11):1173-1185. https://doi.org/10.1101/gad.1915510

Moro E et al (2012) In vivo Wnt signaling tracing through a transgenic biosensor fish reveals novel activity domains. Dev Biol 366(2):327-340. https://doi.org/10.1016/j.ydbio.2012.03.023

Murphy L et al (2008) Lifetime risk of symptomatic knee osteoarthritis. Arthritis Care Res 59(9):1207-1213. https://doi.org/10.1002/ art. 24021

Nakamura Y et al (2012) Sox9 is upstream of microRNA-140 in cartilage. Appl Biochem Biotechnol 166(1):64-71. https://doi. org/10.1007/s12010-011-9404-y

Newham E et al (2019) Finite element and deformation analyses predict pattern of bone failure in loaded zebrafish spines. J R Soc Interface 16(160):20190430. https://doi.org/10.1098/rsif.2019.0430

Nomura $M$ et al (2017) Thinning of articular cartilage after joint unloading or immobilization. An experimental investigation of the pathogenesis in mice. Osteoarthritis Cartilage 25(5):727736. https://doi.org/10.1016/j.joca.2016.11.013

O'Brien J et al (2018) Overview of microRNA biogenesis, mechanisms of actions, and circulation. Front Endocrinol. https://doi. org/10.3389/fendo.2018.00402

Ofer L et al (2019) A novel nonosteocytic regulatory mechanism of bone modeling. PLoS Biol 17(2):e3000140. https://doi. org/10.1371/journal.pbio.3000140

Okamura N et al (2010) Deficiency of tenascin-C delays articular cartilage repair in mice. Osteoarthritis Cartilage 18(6):839-848. https ://doi.org/10.1016/j.joca.2009.08.013

Otterness IG et al (1998) Exercise protects against articular cartilage degeneration in the hamster. Arthritis Rheum 41(11):2068-2076. https://doi.org/10.1002/1529-0131(199811)41:11<2068:AIDART23>3.0.CO;2-L

Palmoski MJ, Colyer RA, Brandt KD (1980) Joint motion in the absence of normal loading does not maintain normal articular cartilage. Arthritis Rheum 23(3):325-334. https://doi. org/10.1002/art.1780230310
Palmoski M, Perricone E, Brandt KD (1979) Development and reversal of a proteoglycan aggregation defect in normal canine knee cartilage after immobilization. Arthritis Rheum 22(5):508-517. https://doi.org/10.1002/art.1780220511

Patwari P et al (2003) Proteoglycan degradation after injurious compression of bovine and human articular cartilage in vitro: interaction with exogenous cytokines. Arthritis Rheum 48(5):12921301. https://doi.org/10.1002/art.10892

Pond MJ, Nuki G (1973) Experimentally-induced osteoarthritis in the dog. Ann Rheum Dis 32(4):387-388. https://doi.org/10.1136/ ard.32.4.387

Poulet B et al (2011) Characterizing a novel and adjustable noninvasive murine joint loading model. Arthritis Rheum 63(1):137-147. https://doi.org/10.1002/art.27765

Roberts SR et al (2001) Mechanical compression influences intracellular $\mathrm{Ca} 2+$ signaling in chondrocytes seeded in agarose constructs. J Appl Physiol 90(4):1385-1391. https://doi. org/10.1152/jappl.2001.90.4.1385

Russell W, Burch R (1959) The principles of humane experimental technique. Methuen \& Co., London

Russo CR (2009) The effects of exercise on bone basic concepts and implications for the prevention of fractures. Clin Cases Miner Bone Metab 6(3):223-228

Schilling TF, Kimmel CB (1994) Segment and cell type lineage restrictions during pharyngeal arch development in the zebrafish embryo. Development 120(3):483-494

Schmid TM et al (1991) Late events in chondrocyte differentiation: hypertrophy, type $\mathrm{X}$ collagen synthesis and matrix calcification. In Vivo 5(5):533-540

Shao J et al (2020) MiR-146a-5p promotes IL-1 $\beta$-induced chondrocyte apoptosis through the TRAF6-mediated NF-kB pathway. Inflamm Res 69(6):619-630. https://doi.org/10.1007/s0001 1-020-01346-w

Shapiro F, Glimcher MJ (1980) Induction of osteoarthrosis in the rabbit knee joint. Clin Orthop Relat Res 147:287-295

Sharma G, Saxena RK, Mishra P (2007) Differential effects of cyclic and static pressure on biochemical and morphological properties of chondrocytes from articular cartilage. Clin Biomech 22(2):248-255. https://doi.org/10.1016/j.clinbiomec h.2006.09.008

Shelton JC, Bader DL, Lee DA (2003) Mechanical conditioning influences the metabolic response of cell-seeded constructs. Cells Tissues Organs 175(3):140-150. https://doi. org/10.1159/000074630

Shwartz Y et al (2012) Muscle contraction controls skeletal morphogenesis through regulation of chondrocyte convergent extension. Dev Biol 370(1):154-163. https://doi.org/10.1016/j.ydbio 2012.07.026

Millward-Sadler SJ et al (2000) Mechanotransduction via integrins and interleukin- 4 results in altered aggrecan and matrix metalloproteinase 3 gene expression in normal, but not osteoarthritic, human articular chondrocytes. Arthritis Rheu 43(9):2091-2099. https://doi.org/10.1002/1529-0131(200009)43:9<2091:AIDANR21>3.0.CO;2-C

Soltz MA et al (2000) Functional tissue engineering of articular cartilage through dynamic loading of chondrocyte-seeded agarose gels. J Biomech Eng 122(3):252. https://doi.org/10.1115/1.42965 6

Song J et al (2015) MicroRNA-222 regulates MMP-13 via targeting HDAC-4 during osteoarthritis pathogenesis. BBA Clin 3(1):7989. https://doi.org/10.1016/j.bbacli.2014.11.009

Sood SC (1971) A study of the effects of experimental immobilisation on rabbit articular cartilage. J Anat 108(Pt 3):497-507

Swingler TE et al (2012) The expression and function of microRNAs in chondrogenesis and osteoarthritis. Arthritis Rheum 64(6):1909_ 1919. https://doi.org/10.1002/art.34314 
Taylor JS et al (2003) Genome duplication, a trait shared by 22,000 species of ray-finned fish. Genome Res 13(3):382-390. https:// doi.org/10.1101/gr.640303

Tomiya M et al (2009) Skeletal unloading induces a full-thickness patellar cartilage defect with increase of urinary collagen II CTx degradation marker in growing rats. Bone 44(2):295-305. https ://doi.org/10.1016/j.bone.2008.10.038

Tuddenham L et al (2006) The cartilage specific microRNA-140 targets histone deacetylase 4 in mouse cells. FEBS Lett 580(17):42144217. https://doi.org/10.1016/j.febslet.2006.06.080

Wada $\mathrm{N}$ et al (2005) Hedgehog signaling is required for cranial neural crest morphogenesis and chondrogenesis at the midline in the zebrafish skull. Development 132(17):3977-3988. https://doi. org/10.1242/dev.01943

Weiner AMJ et al (2019) Dicer1 is required for pigment cell and craniofacial development in zebrafish. Biochimic Biophys Acta Gene Regul Mech 4:472-485. https://doi.org/10.1016/j.bbagr m.2019.02.005

Wellcome Trust (2013) Zebrafish genome yields significant similarity to human genome I Wellcome.

Wienholds E et al (2003) The microRNA-producing enzyme Dicer1 is essential for zebrafish development. Nat Genet 35(3):217-218. https://doi.org/10.1038/ng1251
Wienholds E et al (2005) Cell biology: microRNA expression in zebrafish embryonic development. Science 309(5732):310-311. https://doi.org/10.1126/science.1114519

Wopat S et al (2018) Spine patterning is guided by segmentation of the notochord sheath. Cell Rep 22(8):2026-2038. https://doi. org/10.1016/j.celrep.2018.01.084

Wu X et al (2019) MiR-27a targets DKK2 and SFRP1 to promote reosseointegration in the regenerative treatment of peri-implantitis. J Bone Miner Res 34(1):123-134. https://doi.org/10.1002/ jbmr.3575

Yang X et al (2016) Mechanical and IL-1 $\beta$ responsive miR-365 contributes to osteoarthritis development by targeting histone deacetylase 4. Int J Mol Sci 17(4):436. https://doi.org/10.3390/ijms1 7040436

Publisher's Note Springer Nature remains neutral with regard to jurisdictional claims in published maps and institutional affiliations. 